\title{
Chapter 45 \\ Report on Global Environment \\ Competitiveness of Czech Republic
}

Czech is a landlocked country in Central Europe. The country is bordered by Poland to the north, Germany to the west, Austria to the south and Slovakia to the east. The Czech Republic has a temperate continental climate, with relatively hot summers and cold, cloudy and snowy winters. It covers 77.3 thousand of square kilometers and has a

\begin{tabular}{|l|}
\hline Score: \\
51.68 \\
\hline Rank: \\
47 \\
\hline
\end{tabular}
population of 10.50 million. Its GDP reaches $\$ 217.03$ billion in 2011 . Through the index system and evaluation model, the environment competitiveness index of Czech Republic ranks at 47 in 133 countries.

Fig. 45.1 Contribution

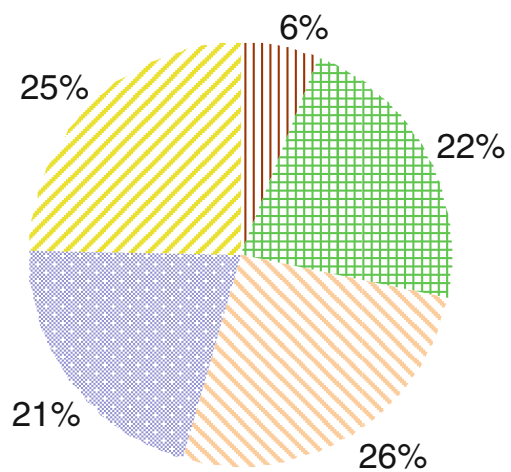

III Resource Environment Competitiveness

\# Ecological Environment Competitiveness Environment Carrying Competitiveness

- Environment Management Competitiveness

Environment Harmony of sub-index to GEC Competitiveness 


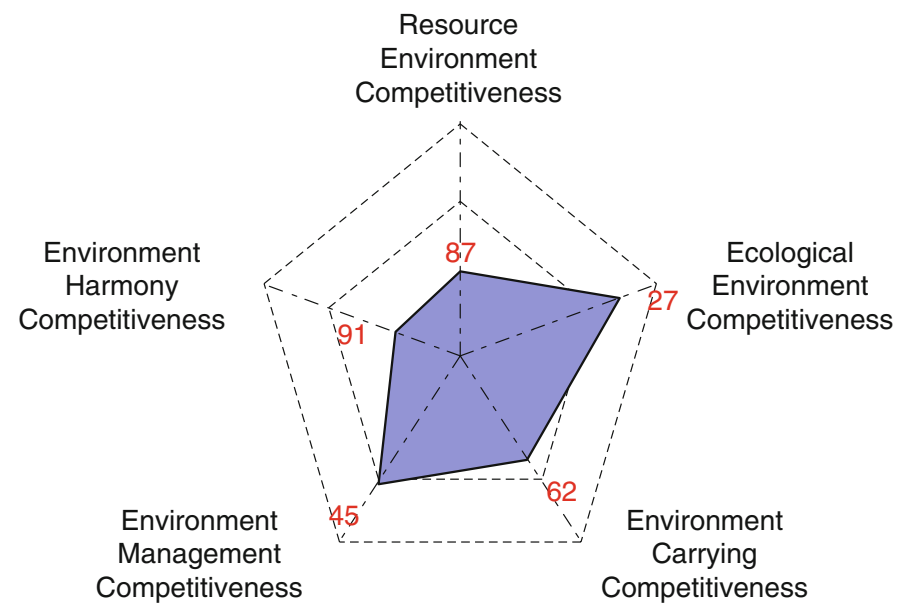

Fig. 45.2 Rank of sub-index of GEC

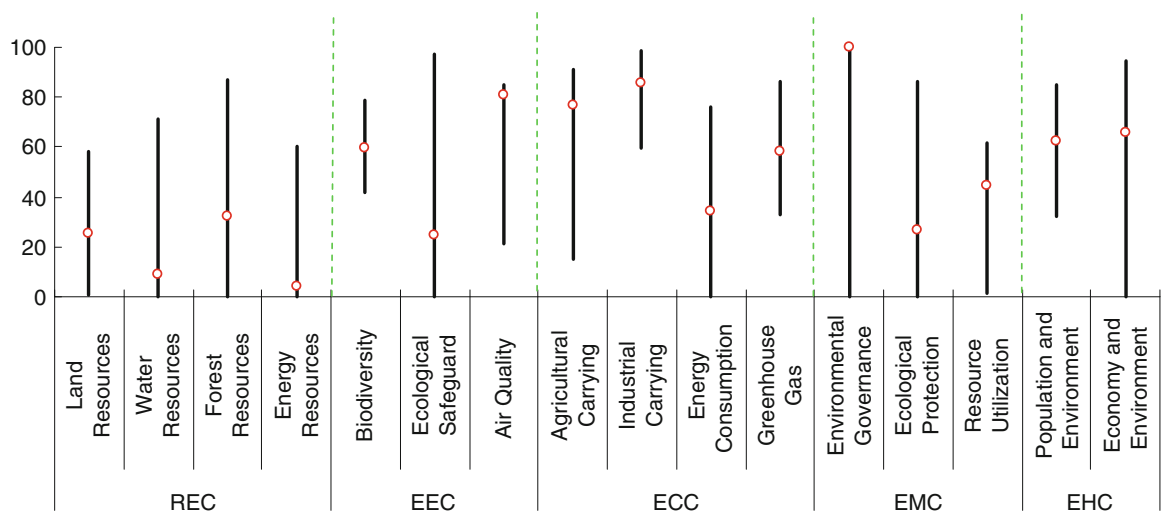

Fig. 45.3 Score and rank of the pillars of GEC

Table 45.1 Score and rank of all indicators of GEC

\begin{tabular}{|c|c|c|c|c|c|}
\hline Indicators & Score & Rank & Indicators & Score & Rank \\
\hline 1 Resource Environment & 15.57 & 87 & Groundwater & 2.63 & 98 \\
\hline Competitiveness & & & Total internal renewable water & 8.59 & 85 \\
\hline 1.1 Land Resources & 25.60 & 16 & resources & & \\
\hline Land area per capita & 1.30 & 103 & 1.3 Forest Resources & 32.36 & 56 \\
\hline $\begin{array}{l}\text { Percentage of arable land to } \\
\text { total land area }\end{array}$ & 69.50 & 8 & $\begin{array}{l}\text { Growing stock in forest and } \\
\text { other wooded land }\end{array}$ & 52.33 & 47 \\
\hline Arable land per capita & 14.11 & 34 & Proportion of land area & 40.32 & 46 \\
\hline 1.2 Water Resources & 9.12 & 96 & covered by forest & & \\
\hline Surface water & 1.84 & 95 & Forest area per capita & 1.77 & 66 \\
\hline Annual precipitation & 23.43 & 76 & 1.4 Energy Resources & 4.14 & 79 \\
\hline
\end{tabular}


Table 45.1 (continued)

\begin{tabular}{|c|c|c|c|c|c|}
\hline Indicators & Score & Rank & Indicators & Score & Rank \\
\hline Fossil energy & 1.43 & 30 & 3.3 Energy Consumption & 34.22 & 58 \\
\hline Energy production & 3.04 & 32 & Energy consumption per unit & 98.78 & 111 \\
\hline Proportion of combustible & 6.44 & 72 & of land area & & \\
\hline $\begin{array}{l}\text { renewable and waste to } \\
\text { total energy consumption }\end{array}$ & & & $\begin{array}{l}\text { Ratio of clean energy } \\
\text { consumption }\end{array}$ & 29.91 & 23 \\
\hline $\begin{array}{l}\text { Net energy imports of the } \\
\text { energy consumption }\end{array}$ & 9.05 & 68 & $\begin{array}{l}\text { Elasticity of energy } \\
\text { consumption }\end{array}$ & 8.19 & 125 \\
\hline $\begin{array}{l}2 \text { Ecological Environment } \\
\text { Competitiveness }\end{array}$ & 57.61 & 27 & $\begin{array}{l}\text { Elasticity of electric power } \\
\text { consumption }\end{array}$ & 0.00 & 124 \\
\hline 2.1 Biodiversity & 59.54 & 22 & 3.4 Greenhouse Gas & 58.07 & 98 \\
\hline threatened fish species & 99.06 & 6 & Growth rate of $\mathrm{CO}_{2}$ & 51.67 & 67 \\
\hline threatened mammal species & 98.91 & 6 & emissions & & \\
\hline threatened plant species & 99.53 & 56 & Growth rate of Methane & 58.95 & 68 \\
\hline $\begin{array}{l}\text { GEF benefits index for } \\
\text { biodiversity }\end{array}$ & 0.10 & 122 & $\begin{array}{l}\text { emissions } \\
\mathrm{CO}_{2} \text { emissions per unit }\end{array}$ & 98.35 & 118 \\
\hline 2.2 Ecological Safeguard & 24.46 & 59 & of land area & 30.53 & 110 \\
\hline Terrestrial protected areas & 40.76 & 44 & $\mathrm{CO}_{2}$ emissions per unit of & 29.71 & 102 \\
\hline Marine protected areas & N/A & N/A & energy consumption & & \\
\hline 2.3 Air Quality & 81.03 & 19 & 4 Environment Management & 54.00 & 45 \\
\hline Inhalable particles (PM10) & 88.32 & 23 & Competitiv & & \\
\hline Particulate matter (PM2.5) & 86.26 & 61 & 4.1 Environmental Governance & 100.00 & 1 \\
\hline Index of indoor air pollution & 100.00 & 1 & $\begin{array}{l}\text { Agricultural chemicals } \\
\text { regulation }\end{array}$ & 100.00 & 1 \\
\hline Nitrogen oxides emission & 67.11 & 83 & regulation & & \\
\hline Sulfur dioxide emission & 40.33 & 83 & centage of the rural & 100.00 & 1 \\
\hline $\begin{array}{l}3 \text { Environment Carrying } \\
\text { Competitiveness }\end{array}$ & 67.19 & 62 & an improved water source & & \\
\hline 3.1 Agricultural Carrying & 76.51 & 21 & $\begin{array}{l}\text { Percentage of the urban } \\
\text { population with access to }\end{array}$ & 100.00 & 1 \\
\hline $\begin{array}{l}\text { Cereal yield per unit of arable } \\
\text { land }\end{array}$ & 48.75 & 31 & an improved water source & & \\
\hline $\begin{array}{l}\text { Fertilizer consumption per unit } \\
\quad \text { of arable land }\end{array}$ & 90.03 & 85 & $\begin{array}{l}\text { 4.2 Ecological Protection } \\
\text { Area of plantation and } \\
\text { afforestation }\end{array}$ & $\begin{array}{r}26.60 \\
3.42\end{array}$ & $\begin{array}{l}90 \\
18\end{array}$ \\
\hline Annual freshwater & 99.99 & 4 & Biome protect & 84.10 & 38 \\
\hline $\begin{array}{l}\text { withdrawals for agriculture } \\
\text { per unit of arable land }\end{array}$ & & & $\begin{array}{l}\text { Overfishing of fishing } \\
\text { resources }\end{array}$ & N/A & N/A \\
\hline 3.2 Industrial Carrying & 85.95 & 106 & 4.3 Resource Utilization & 44.55 & 31 \\
\hline $\begin{array}{l}\text { Net exports of goods as a } \\
\text { percentage of GDP }\end{array}$ & 52.57 & 123 & $\begin{array}{l}\text { Utilization rate of water } \\
\text { resources }\end{array}$ & 0.60 & 53 \\
\hline $\begin{array}{l}\text { Electric power consumption } \\
\text { per unit of value added of } \\
\text { industry }\end{array}$ & 92.89 & 55 & $\begin{array}{l}\text { Percentage of total internal } \\
\text { renewable water resources } \\
\text { to total water resources }\end{array}$ & 90.19 & 14 \\
\hline $\begin{array}{l}\mathrm{SO}_{2} \text { emissions per unit of } \\
\text { value added of industry }\end{array}$ & 99.91 & 38 & $\begin{array}{l}\text { Percentage of agricultural land } \\
\text { to total land area }\end{array}$ & 64.86 & 42 \\
\hline $\begin{array}{l}\text { Annual freshwater } \\
\text { withdrawals for industry } \\
\text { per value added of industry }\end{array}$ & 98.43 & 53 & $\begin{array}{l}\text { Percentage of fossil fuel } \\
\text { energy consumption to } \\
\text { total energy consumption }\end{array}$ & 22.55 & 71 \\
\hline
\end{tabular}


Table 45.1 (continued)

\begin{tabular}{|c|c|c|c|c|c|}
\hline Indicators & Score & Rank & Indicators & Score & Rank \\
\hline 5 Environment Harmony & 64.01 & 91 & $\mathrm{CO}_{2}$ emissions per capita & 70.58 & 116 \\
\hline Competitiveness & & & Energy consumption per & 67.99 & 107 \\
\hline 5.1 Population and & 62.33 & 113 & capita & & \\
\hline Environment & & & 5.2 Economy and Environment & 65.69 & 62 \\
\hline $\begin{array}{l}\text { Percentage of population with } \\
\text { access to Improved }\end{array}$ & 98.00 & 33 & $\begin{array}{l}\text { Land resource utilization } \\
\text { efficiency }\end{array}$ & 0.82 & 22 \\
\hline sanitation facilities & & & Sulfur dioxide emissions per & 96.45 & 48 \\
\hline Motor vehicles per 1,000 & 40.37 & 106 & unit of GDP & & \\
\hline $\begin{array}{l}\text { people } \\
\text { Renewable internal freshwater }\end{array}$ & 1.52 & 92 & $\begin{array}{l}\text { Carbon dioxide emissions per } \\
\text { unit of GDP }\end{array}$ & 79.50 & 94 \\
\hline $\begin{array}{c}\text { resources per capita } \\
\mathrm{SO}_{2} \text { emissions per capita }\end{array}$ & 90.96 & 91 & $\begin{array}{l}\text { Energy consumption per unit } \\
\text { of GDP }\end{array}$ & 86.00 & 61 \\
\hline
\end{tabular}

Table 45.2 Rank distribution of the individual indicators of GEC

\begin{tabular}{|c|c|c|c|c|c|c|}
\hline Sub-index & $\begin{array}{l}\text { Number of } \\
\text { the individual } \\
\text { indicators }\end{array}$ & $\begin{array}{l}\text { Rank } \\
1-10\end{array}$ & $\begin{array}{l}\text { Rank } \\
11-30\end{array}$ & $\begin{array}{l}\text { Rank } \\
31-60\end{array}$ & $\begin{array}{l}\text { Rank } \\
61-100\end{array}$ & $\begin{array}{l}\text { Rank } \\
101-133\end{array}$ \\
\hline $\begin{array}{l}\text { Resource Environment } \\
\text { Competitiveness }\end{array}$ & 14 & 1 & 2 & 4 & 6 & 1 \\
\hline $\begin{array}{l}\text { Ecological Environment } \\
\text { Competitiveness }\end{array}$ & 11 & 3 & 3 & 3 & 2 & 0 \\
\hline $\begin{array}{l}\text { Environment Carrying } \\
\text { Competitiveness }\end{array}$ & 15 & 0 & 2 & 4 & 4 & 5 \\
\hline $\begin{array}{l}\text { Environment Management } \\
\text { Competitiveness }\end{array}$ & 10 & 3 & 2 & 4 & 1 & 0 \\
\hline $\begin{array}{l}\text { Environment Harmony } \\
\text { Competitiveness }\end{array}$ & 10 & 0 & 1 & 2 & 4 & 3 \\
\hline Total & 60 & 7 & 10 & 17 & 17 & 9 \\
\hline
\end{tabular}

Open Access This chapter is distributed under the terms of the Creative Commons Attribution Noncommercial License, which permits any noncommercial use, distribution, and reproduction in any medium, provided the original author(s) and source are credited. 